\title{
Energy-Efficient Deployment of a Non-Orthogonal Multiple Access Unmanned Aerial System
}

\author{
Nithin Babu ${ }^{1,2}$, Constantinos B. Papadias ${ }^{1,2}$, and Petar Popovski ${ }^{2}$ \\ ${ }^{1}$ Research, Technology and Innovation Network (RTIN), ALBA, The American College of Greece, Greece \\ \{nbabu,cpapadias\}@acg.edu \\ ${ }^{2}$ Department of Electronic Systems, Aalborg University, Denmark \\ $\{$ niba,cop,petarp\}@es.aau.dk
}

\begin{abstract}
In this work, we propose a methodology for the energy-efficient placement of an unmanned aerial system (UAS) deployed to collect data from a set of ground user equipments (UEs). The data-communication between the UEs and the UxNB, a radio access node carried by an unmanned aerial vehicle (UAV), of the UAS follows a non-orthogonal multiple-access (NOMA) scheme; in which all the UEs share the same time and frequency resources. The receiver removes the inter-UE interference from the co-channel UEs through joint implementation of a powerreduction technique at the UEs and the successive interference cancellation (SIC) at the receiver. Firstly, a new energy-efficiency metric, area energy efficiency (AEE) representing the total area covered by a UxNB per Watt-Hour (Wh) of energy consumed, is introduced. Then, the optimal hovering altitude of the UxNB that maximizes the AEE is determined using the proposed algorithm. Numerical evaluations show that the obtained solution using the proposed algorithm matches the globally optimal solution, and the proposed NOMA scheme prevails over an equivalent orthogonal multiple access (OMA) scheme, in terms of the AEE.
\end{abstract}

Index Terms-Area energy-efficiency, UAS placement optimization, NOMA communication, UAV communication.

\section{INTRODUCTION}

Next-generation wireless networks are expected to support high data rates and a larger number of user devices [1]. Standardization bodies like the 3rd generation partnership project (3GPP) has been considering meeting these demands with non-orthogonal multiple access (NOMA) schemes [2]. The NOMA scheme supports users by differentiating them in power or space domains, thereby allowing them to share the same time and frequency resources. The NOMA scheme that separates the users in the power domain, called powerdomain NOMA (P-NOMA), requires a successive interference cancellation (SIC) receiver [4]. The SIC receiver exploits the diversity in the received signal-to-interference-plus-noise ratio (SINR) from the user equipments (UEs): the message from the strongest UE (highest received SINR) is decoded treating all

(C)2021 IEEE. Personal use of this material is permitted. Permission from IEEE must be obtained for all other uses, in any current or future media, including reprinting/republishing this material for advertising or promotional purposes, creating new collective works, for resale or redistribution to servers or lists, or reuse of any copyrighted component of this work in other works. This version of the work has been ACCEPTED for presentation at IEEE ICC 2021-Workshops (WS04). This work is supported by the project PAINLESS which has received funding from the European Union's Horizon 2020 research and innovation programme under grant agreement No 812991. the other UE's messages as noise; then, the message from the second-strongest UE is decoded after subtracting the strongest UE's message from the received signal. This continues until the SIC receiver decodes the message from the UE with the lowest received SINR value.

UAV-assisted networks deployed to provide cellular service for applications having temporary/emergency nature such as frequent data collection from a set of Internet-of-Things (IoT) nodes or assisting the rescue operation during a natural disaster, is considered as one of the promising application of the next-generation wireless networks [3]. Here, we consider such a UAV-assisted network in which the UAV carries a NOMAbased radio access node to the service-required area. The latest 3 GPP report, [15], has enlisted various technical specifications of a UAV-assisted network.

The concept of a UAV-assisted NOMA system has been considered in the works [5]- [14]. [4] presents a survey on the existing studies that have considered the integration of the P-NOMA scheme with the enabling communications schemes and technologies, which are expected to meet the various requirements of next-generation wireless networks. In [5], the authors study the P-NOMA scheme for the energyefficient placement of a UAV- base station using the userpairing method. The individual users in a pair will be using the same time and frequency resources; whereas, the resources allocated to two different user pairs will be orthogonal. The placement and power allocation that maximize the downlink sum rate of a NOMA-UAV network is proposed in [6]. The work in [7] maximizes the minimum downlink throughput of a UAV network by jointly optimizing multi-user communication scheduling and the UAV trajectory. The joint optimization problem is decoupled into two blocks (the scheduling and the UAV trajectory blocks) and is solved using the block coordinate descent method. The authors of [8], propose a solution to the max-min rate optimization problem of a UAV-enabled NOMA communication system. The optimization problem is formulated subject to total power, bandwidth, altitude, and beamwidth constraints, and is solved using the sequential convex programming technique. In [9], the authors present a joint UAV trajectory design and resource allocation algorithm that maximizes the minimum average rate among the ground users of a UAV communication system. The formulated max- 
min downlink data rate problem is solved using the penalty dual decomposition method. The work in [10] minimizes the transmit power of a UAV-NOMA system subject to the minimum achievable rate requirements; whereas, the sum rate a similar system is maximized in [11] by jointly optimizing the UAV trajectory and the NOMA precoding. The authors of [12] aim to maximize the system capacity of a UAV-NOMA system by jointly optimizing the subchannel assignment, the uplink transmit power, and the flying heights of the UAVs using the $\mathrm{K}$-means clustering method and the matching theory. [13] considers a different application scenario in which a UAV system co-exists with a set of ground users; the authors maximize both the data rate from the UAV to the base stations and the data rate of the co-channel ground users to their associated base stations by optimizing the precoding vectors at the multiantenna UAV. [14] discusses the possibility of using a UAV as a full-duplex relay to aid the communication between a base station and two NOMA users. Considering a simultaneous wireless information and power transfer technique, the authors aim at maximizing the sum throughput of the whole system and the harvested energy at the UAV based on the inner approximation method.

The works in [5]- [10] consider a downlink-NOMA communication between a single- or multi- UAV system and a set of ground users. Except [5], none of the above works maximize the energy-efficiency of a NOMA-UAV system considering both the communication and the UAV energy consumption. Additionally, the user-pairing method considered in [5] exploits only partial non-orthogonality (between two user pairs) to maximize the number of bits transmitted per Joule of energy consumed. However, [12] and [13] consider an uplink data transmission between a set of users and a UAV, but the energy-efficiency aspect remains unstudied in these works. Being an energy-limited system, we believe that the energyefficient placement of a UAS is of paramount importance. In our previous works, [18] and [19], we have considered the energy-efficient placement of a single- and multi-UAV system, respectively. [18] considers a downlink OMA scheme, whereas in [19], we consider an uplink OMA scheme with the universal frequency reuse among the UAVs. The placement optimization of a NOMA-UAS that maximizes the proposed area energy efficiency (AEE) metric considering both the communication and the UAV energy consumption has, to the best of our knowledge, not been considered in the literature.

Section II explains the system architecture and the assumptions alongside the definitions of the proposed AEE metric and the power-reduction scheme. Our objective here is to find an energy-efficient hovering altitude for the UxNB part of a UAS considering an uplink P-NOMA scheme. As the altitude increases, the area covered by the UxNB increases, the time taken by the ground users to complete the uplink data transmission increases, and the power consumed by the UAV increases. Intending to capture these effects, we introduce a new energy-efficiency metric in Section II-C, the AEE. The AEE of a UAS is neither maximum at a low altitude value nor at a high altitude value. In Section III, we determine the area

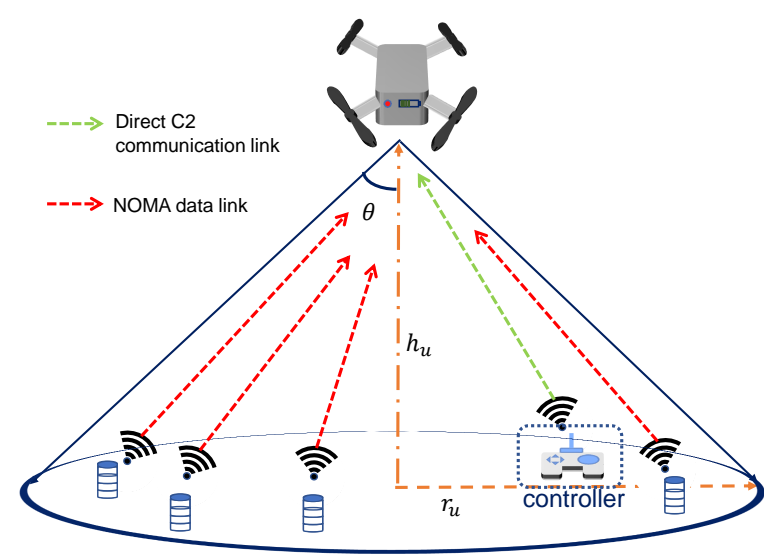

Fig. 1: Unmanned Aerial System Architecture.

energy-efficient hovering altitude for a NOMA-UAS. All our main findings from the numerical evaluations are discussed in Section IV.

\section{System Model And Definitions}

We consider a UxNB, a radio access node carried in the air by a UAV, deployed to collect independent data from a set of uniformly distributed ground UEs with a density $\rho_{u}$. The UxNB with the ground controller, as shown in Fig. 1, forms the UAS model proposed in the latest 3GPP report [15]. The UxNB is assumed to be equipped with a directional antenna of half-power beamwidth $2 \theta$ with antenna gain in direction $(\psi, \omega)$ given by [20],

$$
g_{\mathrm{u}, \mathrm{a}}=\left\{\begin{array}{cc}
\frac{g_{\mathrm{o}, \mathrm{a}}}{\theta^{2}} & -\theta \leq \psi \leq \theta,-\theta \leq \omega \leq \theta, \\
\approx 0 & \text { otherwise },
\end{array}\right.
$$

where $g_{\mathrm{o}, \mathrm{a}} \approx 2.2846$. All the UEs are equipped with an omnidirectional antenna. Also, the UEs send their collected data to the UxNB using the same time and frequency resources, but with different power levels, thereby forming an uplink P-NOMA scheme. The transmitted data packets are successfully decoded using the successive interference cancellation technique at the UxNB. In practice, this maps to the data-collection phase of an IoT network.

\section{A. Propagation Channel}

The ground-to-air channel between a UE and the UxNB falls either to the line-of-sight $(\mathrm{LoS})$ or the non-LoS group depending on its relative position to the UxNB. The LoS probability of a UE-UxNB channel link is expressed as [21],

$$
P_{1}\left(\phi_{i}\right)=\frac{1}{\left.\left\{1+a \exp \left[-b\left(\phi_{i}-a\right)\right)\right]\right\}},
$$

where $\phi_{i}=(180 / \pi) \tan ^{-1}\left(h_{\mathrm{u}} / r_{i}\right)$ is the elevation angle between the $i^{\text {th }}$ UE located at a distance of $r_{i}$ from the center of the UxNB's coverage area, and the UxNB hovering at an altitude $h_{\mathrm{u}} ; a, b$ are the environment-dependent parameters that 
depend on the building profile of the deploying area. Hence, the probabilistic mean path loss is given by [19],

$$
\begin{aligned}
\bar{L}_{i}\left(h_{\mathrm{u}}\right) & =P_{1}\left(\phi_{i}\right) \times L_{1, i}+P_{2}\left(\phi_{i}\right) \times L_{2, i}, \\
& =\frac{\left(r_{i}^{2}+h_{\mathrm{u}}^{2}\right)}{h_{\mathrm{o}}}\left\{P_{1}\left(\phi_{i}\right) \mu_{1}^{2}+\left[1-P_{1}\left(\phi_{i}\right)\right] \mu_{2}^{2}\right\},
\end{aligned}
$$

where, $\mu_{1}^{2}$ and $\mu_{2}^{2}$ are the mean values of the additional path loss due to the long-term random channel variations associated with the respective LoS and NLoS links; $h_{\mathrm{O}}$ is the channel gain at a reference distance of $1 \mathrm{~m}$.

\section{B. Uplink P-NOMA Scheme}

The uplink power control mechanism in LTE systems require that the received powers from different UEs equal the same target power, $p_{\mathrm{u}}$ [22]. Hence, the power transmitted by a UE assuming full channel-loss compensation is given by,

$$
p\left(h_{\mathrm{u}}\right)=p_{\mathrm{u}} \bar{L}_{i}\left(h_{\mathrm{u}}\right) .
$$

For successful decoding of the received messages in the presence of co-channel interference, the SIC receiver at the UxNB requires different received powers per UE. First of all, the UEs send individual control messages to the UxNB with the same transmit power using orthogonal control channels. The UxNB then assigns indices of the decoding order to the UEs based on the corresponding path loss values: a higher index value to a UE with a higher path loss value. That is, in an instance with $N$ UEs in the coverage region of the $\mathrm{UxNB}$, the closest $\mathrm{UE}$ to the center of the coverage region is assigned the index 1 , whereas the farthest UE the index $N$. Next, the UxNB informs the UEs of their corresponding index of the decoding order through the associated control channels. Now, the UEs follow a power-reduction scheme for the concurrent transmission of the messages using the same frequency resource. Accordingly, the UE with the index of decoding order $i$ transmits its message with a power given by [16],

$$
p_{i}\left(h_{\mathrm{u}}\right)=\frac{p_{\mathrm{u}} \bar{L}_{i}\left(h_{\mathrm{u}}\right)}{\delta^{i-1}},
$$

where, $\delta$ is the power-reduction factor. (6) guarantees the required power diversity at the SIC receiver of the UxNB unit. Consider the UxNB hovering at an altitude $h_{\mathrm{u}}$ covering $N\left(h_{\mathrm{u}}\right)=\rho_{\mathrm{u}} \pi h_{\mathrm{u}}^{2} \tan ^{2} \theta$ uniformly distributed UEs. Then, the received signal at the UxNB, from the UEs transmitting the messages with the P-NOMA scheme, is expressed as,

$$
y_{\mathrm{u}}=\sum_{i=1}^{N\left(h_{\mathrm{u}}\right)} \sqrt{\frac{p_{i}\left(h_{\mathrm{u}}\right)}{\bar{L}_{i}\left(h_{\mathrm{u}}\right)}} x_{i}+\sigma_{\mathrm{n}}^{2},
$$

where, $x_{i}$ is the message transmitted by the $i^{\text {th }} \mathrm{UE}$, and $\sigma_{\mathrm{n}}^{2}=$ $\sigma_{0}^{2} W$ is the zero-mean additive white Gaussian noise power with a power spectral density $\sigma_{0}^{2}$ over a channel bandwidth $W$. Using the diverse received power from the UEs, the SIC receiver starts decoding the message from the first UE (the UE with the index 1) considering the messages from the remaining $\left(N\left(h_{\mathrm{u}}\right)-1\right)$ UEs as co-channel interference. Correspondingly, the $i^{\text {th }}$ UE's message is decoded only after decoding the prior $(i-1)$ UEs' messages; hence, the received SINR value from the $i^{\text {th }} \mathrm{UE}$ is given by,

$$
\Gamma_{i}^{\mathrm{n}}\left(h_{\mathrm{u}}\right)=\frac{\frac{p_{i}\left(h_{\mathrm{u}}\right)}{\bar{L}_{i}\left(h_{\mathrm{u}}\right)}}{\sum_{j=i+1}^{N\left(h_{\mathrm{u}}\right)} \frac{p_{j}\left(h_{\mathrm{u}}\right)}{\bar{L}_{j}\left(h_{\mathrm{u}}\right)}+\sigma_{\mathrm{n}}^{2}} .
$$

(8) assumes successful decoding of the messages from the first to the $(i-1)^{\text {th }} \mathrm{UE}$; an additional noise term in the denominator of $\Gamma_{i}^{\mathrm{n}}\left(h_{\mathrm{u}}\right)$ represents the co-channel interference from the remaining $\left(N\left(h_{\mathrm{u}}\right)-i\right)$ UEs. In Section III, we incorporate the above-mentioned condition as quality-of-service (QoS) constraints: $\Gamma_{i}^{\mathrm{n}}\left(h_{\mathrm{u}}\right) \geq \Gamma_{\mathrm{m}} \forall i \in \mathcal{N}=\left\{1,2, \ldots, N\left(h_{\mathrm{u}}\right)\right\}$; $\Gamma_{\mathrm{m}}$ is the minimum SINR required at the receiver for the successful decoding of the message from a UE. Therefore, the achievable data rate of the $i^{\text {th }} \mathrm{UE}$ in bits-per-second (bps) assuming perfect channel modulation, coding scheme, and known channel state information (CSI) at the transmitter is given by (9):

$$
R_{i}^{\mathrm{n}}\left(h_{\mathrm{u}}\right)=W \log _{2}\left[1+\Gamma_{i}^{\mathrm{n}}\left(h_{\mathrm{u}}\right)\right] \forall i \in \mathcal{N} .
$$

\section{Area Energy Efficiency}

We define the area energy-efficiency of a UAS as the ratio between the geographical area covered by the UAS, and the total energy consumption:

$$
\operatorname{AEE}_{\mathrm{n}}\left(h_{\mathrm{u}}\right)\left[\mathrm{m}^{2} / \mathrm{Wh}\right]=\frac{\pi r_{\mathrm{u}}^{2}\left(h_{\mathrm{u}}\right)\left[\mathrm{m}^{2}\right]}{T_{\mathrm{u}} P_{\mathrm{t}}\left(h_{\mathrm{u}}\right) \kappa[\mathrm{Wh}]},
$$

where, $P_{\mathrm{t}}\left(h_{\mathrm{u}}\right)=P_{\mathrm{u}}\left(h_{\mathrm{u}}\right)+P_{\mathrm{c}}$ is the total power consumed by the UxNB in Watts with $P_{\mathrm{u}}\left(h_{\mathrm{u}}\right)=\alpha_{\mathrm{u}} h_{\mathrm{u}}+\beta_{\mathrm{u}}$, the power consumed by the UAV while hovering at an altitude $h_{\mathrm{u}}$, and $P_{\mathrm{c}}$ is the communication-related power consumed by the radioaccess node; $T_{\mathrm{u}}$ is the time during which the UxNB remains aloft; $\kappa=2.78 \times 10^{-} 4$ is the Joule to Wh conversion factor. Furthermore, the UAV's power consumption is modelled as an increasing function of the hovering altitude to capture the effect of increased air pressure and temperature, which demands the UAV to generate more power to maintain a stable hovering position at higher altitudes [17], [19]; $\alpha_{\mathrm{u}}$, $\beta_{\mathrm{u}}$ are the UAV-dependent parameters obtained through the empirical study on the UAV's hovering energy consumption reported in [17]; $r_{\mathrm{u}}\left(h_{\mathrm{u}}\right)=h_{\mathrm{u}} \tan \theta$ is the radius of the UxNB's circular coverage region. The AEE, defined in (10), represents the trade-off between an increase in the coverage area and the associated increase in the total energy spent to cover the area. Since available energy-efficiency metrics like the global energy efficiency (GEE), which represents the number of bits transmitted per Joule of energy consumed, do not capture this trade-off, we believe that the proposed AEE metric is well suited for energy-efficient placement of a UAS deployed for rural applications, where the coverage area and the energy consumption factors are of great importance.

\section{OPtimal HOVERING ALTITUdE OF THE UXNB}

In this section, we determine the hovering altitude of the UxNB that maximizes the AEE subject to the altitude 
and minimum QoS constraints. All the UEs follow the PNOMA scheme to transmit $D$ bits of data to the UxNB. The corresponding optimization problem can be written as;

$$
\begin{array}{ll}
\text { (P1) }: \underset{h_{\mathrm{u}}}{\operatorname{maximize}} \frac{\pi h_{\mathrm{u}}^{2} \tan ^{2} \theta}{T_{\mathrm{u}}^{\mathrm{n}}\left(h_{\mathrm{u}}\right) P_{t}\left(h_{\mathrm{u}}\right) \kappa}, \\
\text { s.t. } & h_{\min } \leq h_{\mathrm{u}} \leq h_{\mathrm{max}}, \\
& \Gamma_{i}^{\mathrm{n}}\left(h_{\mathrm{u}}\right) \geq \Gamma_{\mathrm{m}} \quad \forall i \in \mathcal{N}, \\
& T_{\mathrm{u}}^{\mathrm{n}}\left(h_{\mathrm{u}}\right)=\frac{D}{\min \left\{R_{i}^{\mathrm{n}}\left(h_{\mathrm{u}}\right): \forall i \in \mathcal{N}\right\}} .
\end{array}
$$

The objective function of (P1) is the AEE as defined in (10); (12) is the altitude constraint with $h_{\min }$ and $h_{\max }$, the respective lower and upper limits of the hovering altitude specified by the regulatory board. (13) represents the QoS constraints that ensure the successful decoding of the received messages from the UEs hence validating (8) and (9). (14) represents the maximum time taken among the UEs in the UxNB's circular coverage region, to complete the uplink data transmission of $D$ bits of data. (13) can be equivalently written as,

$$
\operatorname{minimum}\left[\Gamma_{i}^{\mathrm{n}}\left(h_{\mathrm{u}}\right)\right] \geq \Gamma_{\mathrm{m}} \text {. }
$$

From (8), the received SINR from a UE depends on both the received and the interference-plus-noise (IN) power values. Considering the first UE, with an increase in the number of UEs in the coverage region of the UxNB, even though the number of interfering UEs increases, the magnitude of interference from each of the $(N-1)$ UEs decreases; whereas, for the $N^{\text {th }} \mathrm{UE}$, the received power decreases exponentially. Hence, to find minimum $\left[\Gamma_{i}^{\mathrm{n}}\left(h_{\mathrm{u}}\right)\right]$, we propose the UxNB to cover a minimum number of UEs as reported in (16).

Proposition 1: For given $\gamma_{\mathrm{n}}=p_{\mathrm{u}} / \sigma_{\mathrm{n}}^{2}, \rho_{\mathrm{u}}, \delta$, and $\theta$ values, the inequality $\Gamma_{1}^{\mathrm{n}}\left(h_{\mathrm{u}}\right)>\Gamma_{2}^{\mathrm{n}}\left(h_{\mathrm{u}}\right)>\ldots>\Gamma_{N}^{\mathrm{n}}\left(h_{\mathrm{u}}\right)$ is satisfied when the UxNB covers a minimum number of UEs given by (16):

$$
N_{\min }\left(h_{\min }^{\prime}\right)=1+\frac{1}{\Delta} \log _{2}\left(\frac{\gamma_{\mathrm{n}}}{\delta-1}\right),
$$

where $\Delta=\log _{2} \delta$.

Proof: Substituting (6) in (8), the SINR expression of the $i^{\text {th }}$ $\overline{\mathrm{UE}}$ can be rewritten as,

$$
\begin{aligned}
\Gamma_{i}^{\mathrm{n}}\left(h_{\mathrm{u}}\right) & =\frac{\frac{\gamma_{\mathrm{n}}}{\delta^{i-1}}}{\sum_{j=i+1}^{N\left(h_{\mathrm{u}}\right)} \frac{\gamma_{\mathrm{n}}}{\delta^{j-1}}+1}, \\
& =\frac{\gamma_{\mathrm{n}}}{\frac{\gamma_{\mathrm{n}}}{\delta}\left[\frac{1-1 / \delta^{N}\left(h_{\mathrm{u}}\right)-i}{1-1 / \delta}\right]+\delta^{i-1}}
\end{aligned}
$$

From (18), $\Gamma_{1}^{\mathrm{n}}\left(h_{\mathrm{u}}\right)>\Gamma_{N}^{\mathrm{n}}\left(h_{\mathrm{u}}\right)$ is equivalently written as,

$$
\frac{\gamma_{\mathrm{n}}}{\frac{\gamma_{\mathrm{n}}}{\delta}\left[\frac{1-1 / \delta^{N\left(h_{\mathrm{u}}\right)-1}}{1-1 / \delta}\right]+1}>\frac{\gamma_{\mathrm{n}}}{\delta^{N\left(h_{\mathrm{u}}\right)-1}},
$$

rearranging (19) with $N\left(h_{\mathrm{u}}\right)$ on the left-hand side gives (16).

$N\left(h_{\mathrm{u}}\right)=\rho_{\mathrm{u}} \pi h_{\mathrm{u}}^{2} \tan ^{2} \theta$ in (16) gives the minimum hovering altitude of the UxNB to cover $N_{\min }\left(h_{\text {min }}^{\prime}\right)$ UEs as,

$$
h_{\min }^{\prime}=\frac{1}{\tan \theta \sqrt{\rho_{\mathrm{u}} \pi}} \sqrt{1+\frac{1}{\Delta} \log _{2}\left(\frac{\gamma_{\mathrm{n}}}{\delta-1}\right)} .
$$

Using Proposition 1, (13) and (14) are rewritten as (21) and (22), respectively:

$$
\begin{aligned}
& \Gamma_{N}^{\mathrm{n}}\left(h_{\mathrm{u}}\right) \geq \Gamma_{\mathrm{m}}, \\
& T_{\mathrm{u}}^{\mathrm{n}}\left(h_{\mathrm{u}}\right)=\frac{D}{R_{N}^{\mathrm{n}}\left(h_{\mathrm{u}}\right)} .
\end{aligned}
$$

Substituting (6) in (8), and using it in (21) gives,

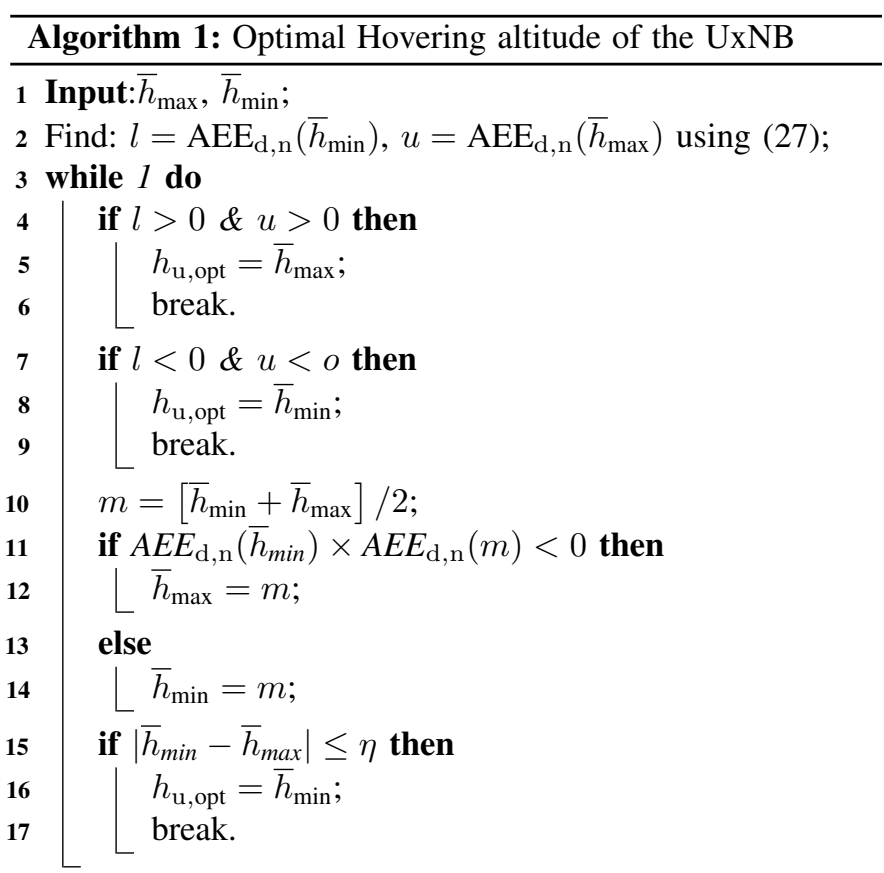

18 Output: Optimal hovering altitude $h_{\mathrm{u}, \mathrm{opt}}$.

$$
\begin{gathered}
\frac{p_{\mathrm{u}}}{\delta^{\left[N\left(h_{\mathrm{u}}\right)-1\right] \sigma_{\mathrm{n}}^{2}} \geq \Gamma_{\mathrm{m}},} \\
N\left(h_{\mathrm{u}}\right) \leq 1+\frac{1}{\Delta} \log _{2}\left(\frac{\gamma_{\mathrm{n}}}{\Gamma_{\mathrm{m}}}\right)=Q\left(\Gamma_{\mathrm{m}}\right) .
\end{gathered}
$$

(24) converts the minimum QoS constraints, (13), into the altitude constraint, $h_{\mathrm{u}} \leq \frac{1}{\tan \theta} \sqrt{\frac{Q\left(\Gamma_{\mathrm{m}}\right)}{\rho_{\mathrm{u}} \pi}}=h_{\max }^{\mathrm{Q}}$. Hence, $(\mathrm{P} 1)$ is reformulated as,

$$
\begin{aligned}
& \text { (P2) : }{\underset{h}{\mathrm{u}}}^{\left.\underset{\operatorname{maximize}}{\operatorname{man}} \frac{D}{R_{N}^{\mathrm{n}}\left(h_{\mathrm{u}}\right)}\right] P_{\mathrm{t}}\left(h_{\mathrm{u}}\right) \kappa}, \\
& \text { s.t. } \underbrace{\max \left\{h_{\min }, h_{\min }^{\prime}\right\}}_{\bar{h}_{\text {min }}} \leq h_{\mathrm{u}} \leq \underbrace{\min \left\{h_{\max }, h_{\max }^{\mathrm{Q}}\right\}}_{\bar{h}_{\max }},
\end{aligned}
$$

Both the numerator and the denominator of the objective function of (P2), $\operatorname{AEE}_{\mathrm{n}}\left(h_{\mathrm{u}}\right)$, are increasing functions of the hovering altitude, $h_{\mathrm{u}}$. The optimal altitude that maximizes $\operatorname{AEE}_{\mathrm{n}}\left(h_{\mathrm{u}}\right)$ is determined using the bisection method as detailed in Algorithm 1.

For a given, $\theta, \rho_{\mathrm{u}}, \gamma_{\mathrm{n}}, \delta$, and $\Gamma_{\mathrm{m}}$ values, the algorithm takes $\bar{h}_{\min }$ and $\bar{h}_{\max }$ as the input parameters. Algorithm 1 makes use of the first derivative property of $\operatorname{AEE}_{\mathrm{n}}\left(h_{\mathrm{u}}\right)$ : 
$\operatorname{AEE}_{\mathrm{d}, \mathrm{n}}\left(h_{\mathrm{u}, \mathrm{opt}}\right)=0 ; \operatorname{AEE}_{\mathrm{d}, \mathrm{n}}\left(h_{\mathrm{u}}\right)>0 \forall h_{\mathrm{u}} \in\left[h_{\min }, h_{\mathrm{u}, \mathrm{opt}}\right) ;$ $\operatorname{AEE}_{\mathrm{d}, \mathrm{n}}\left(h_{\mathrm{u}}\right)<0 \forall h_{\mathrm{u}} \in\left(h_{\mathrm{u}, \mathrm{opt}}, h_{\text {max }}\right]$, where,

$$
\begin{aligned}
\operatorname{AEE}_{\mathrm{d}, \mathrm{n}}\left(h_{\mathrm{u}}\right) & =\frac{W \pi \tan ^{2} \theta}{D \kappa P_{\mathrm{t}}\left(h_{\mathrm{u}}\right)}\left[2 h_{\mathrm{u}} \log _{2}\left(1+\frac{\gamma_{\mathrm{n}}}{\delta^{N\left(h_{\mathrm{u}}\right)-1}}\right)\right] \\
& -\frac{W \pi \tan ^{2} \theta}{D \kappa P_{\mathrm{t}}\left(h_{\mathrm{u}}\right)}\left[\frac{2 h_{\mathrm{u}}^{3} \gamma_{\mathrm{n}} \pi \rho_{\mathrm{u}} \tan ^{2} \theta \ln (\delta)}{\ln (2)\left(\delta^{N\left(h_{\mathrm{u}}\right)-1}+\gamma_{\mathrm{n}}\right)}\right] \\
& -\frac{W \pi \tan ^{2} \theta \alpha_{\mathrm{u}}}{D \kappa P_{\mathrm{t}}^{2}\left(h_{\mathrm{u}}\right)} h_{\mathrm{u}}^{2} \log _{2}\left(1+\frac{\gamma_{\mathrm{n}}}{\delta^{N\left(h_{\mathrm{u}}\right)-1}}\right),
\end{aligned}
$$

and $h_{\mathrm{u}, \mathrm{opt}}$ is the optimal altitude. The algorithm starts with a larger interval supposedly containing the optimal altitude: $h_{\mathrm{u}} \in\left[h_{\min }, \bar{h}_{\max }\right]$. In every iteration, if the altitude corresponding to the midpoint of the interval maps to an $\operatorname{AEE}_{\mathrm{n}}\left(h_{\mathrm{u}}\right)$ value that lies on the falling (rising) edge of the $\operatorname{AEE}_{\mathrm{n}}\left(h_{\mathrm{u}}\right)$ curve, the upper (lower) limit of the interval is changed to the midpoint. This process continues until the interval width is negligible. The interval width after the $k^{\text {th }}$ iteration is $\left(\bar{h}_{\min }-\bar{h}_{\max }\right) / 2^{k}$; therefore, the complexity of the algorithm is $\mathcal{O}\left\{\log _{2}\left[\left(h_{\min }-h_{\max }^{\prime}\right) / \eta\right]\right\}$.

1) OMA-UAS: To compare the AEE performance of the NOMA-UAS with an uplink OMA scheme, we consider that the total available bandwidth, $W$, is equally divided among $N\left(h_{\mathrm{u}}\right)$ UEs. Consequently, the bandwidth allocated to each $\mathrm{UE}$ and the noise power will be a function of the hovering altitude. Thus, for given, $h_{\mathrm{u}}, \rho_{\mathrm{u}}$, and $\theta$ values, the achievable data rate of the $i^{\text {th }}$ UE using the OMA scheme is evaluated using the following expression:

$$
R_{i}^{\mathrm{o}}\left(h_{\mathrm{u}}\right)=\frac{W}{N\left(h_{\mathrm{u}}\right)} \log _{2}\left[1+\frac{\gamma_{\mathrm{n}} N\left(h_{\mathrm{u}}\right)}{\delta^{i-1}}\right] \forall i \in \mathcal{N} .
$$

From (28), it is evident that for a given $h_{\mathrm{u}}$, the inequality $R_{1}^{\mathrm{o}}\left(h_{\mathrm{u}}\right)>R_{2}^{\mathrm{o}}\left(h_{\mathrm{u}}\right)>\ldots>R_{N}^{\mathrm{o}}\left(h_{\mathrm{u}}\right)$ is satisfied. Therefore, the corresponding AEE is expressed as,

$$
\operatorname{AEE}_{\mathrm{o}}\left(h_{\mathrm{u}}\right)=\frac{\pi h_{\mathrm{u}}^{2} \tan ^{2} \theta}{T_{\mathrm{u}}^{\mathrm{o}}\left(h_{\mathrm{u}}\right) P_{t}\left(h_{\mathrm{u}}\right) \kappa},
$$

where $T_{\mathrm{u}}^{\mathrm{o}}\left(h_{\mathrm{u}}\right)=\frac{D}{R_{N}^{\mathrm{o}}\left(h_{\mathrm{u}}\right)}$.

\section{Numerical RESUlts AND Discussion}

In this section, we provide and discuss our main findings obtained through the numerical evaluation. The considered simulation parameters are $h_{0}=1.42 \times 10^{-4}, \mu_{1}=1.0116$, $\mu_{2}=11.2202, a=4.88, b=0.43[19], W=80 \mathrm{MHz}$, $h_{\max }=300 \mathrm{~m}, h_{\min }=10 \mathrm{~m}, \Gamma_{\mathrm{m}}=8.668 \times 10^{-} 4(100 \mathrm{Kbps})$, $D=10^{10}$ bits, $\alpha_{\mathrm{u}}=4.917$, and $\beta_{\mathrm{u}}=275.204$ [17], $\eta=0.5$, $\theta=45^{\circ}, P_{\mathrm{c}}=10 \mathrm{~W}$.

Fig. 2 shows the variations of the SINR values of the first, the middle, and the last UEs, with regards to the hovering altitude of the UxNB. As proved in Proposition 1, in the figure, the SINR value of the last UE (with the index $N$ ) is greater than the first UE (with the index 1) up to the altitude value equal to the right-hand side of (20), beyond which the inequality, $\Gamma_{1}^{\mathrm{n}}\left(h_{\mathrm{u}}\right)>\Gamma_{N}^{\mathrm{n}}\left(h_{\mathrm{u}}\right)$, is satisfied. Moreover, the $h_{\text {min }}^{\prime}$ increases with the signal-to-noise (SNR) ratio value, $\gamma_{n}$, to guarantee a positive difference between the SINR values of

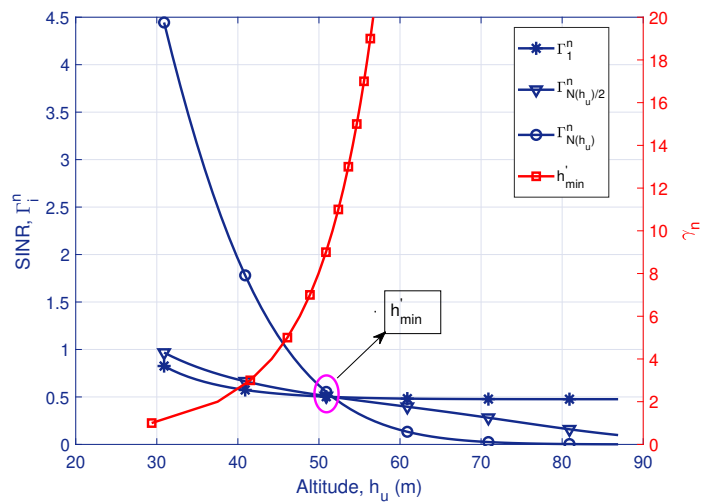

Fig. 2: SINR vs Altitude for $\rho_{\mathrm{u}}=10^{-} 3, \delta=1.5, \gamma_{\mathrm{n}}=10$.

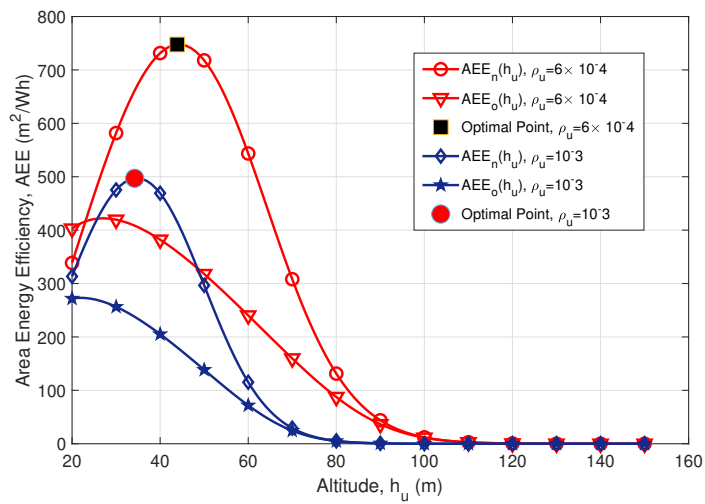

Fig. 3: AEE versus Altitude: $\delta=1.5, \gamma_{\mathrm{n}}=10$.

the first and the last UEs. Fig. 3 shows the variations of the achievable AEE values with the underlying P-NOMA and OMA schemes, plotted using (25) and (29), respectively. The plots are shown against the hovering altitude of the UxNB for $\delta=1.5$ and $\gamma_{\mathrm{n}}=10$. As expected, the AEE is neither maximum at a lower altitude value nor at a higher altitude value. In the low-altitude regime, the smaller area covered by the UxNB (the numerator of the AEE) overcompensates the reduced UxNB's energy consumption resulting in a low AEE value. Similarly, in the high-altitude regime, the UxNB takes more time to complete the uplink data transmission from the UEs demanding a longer UAV endurance; hence, the increased UAV energy consumption at a higher altitude reduces the AEE value despite the increased coverage area. As shown in the figure, the combined effect of the above-mentioned trade offs makes the AEE function a bell-shaped curve. Also, the optimal points obtained through Algorithm 1, are the global optima of the AEE plots. Furthermore, the figure shows that the maximum AEE value considering the NOMA scheme is always higher than the corresponding value obtained using the OMA scheme.

The effect of the UE density over the maximum AEE value is shown in Fig. 4. For both the NOMA and the OMA schemes, the respective maximum AEE values decrease with an increase in the UE density: the $T_{\mathrm{u}}\left(h_{\mathrm{u}}\right)$ factor increases because of the decrease in the received SINR from the edge-UE owing 


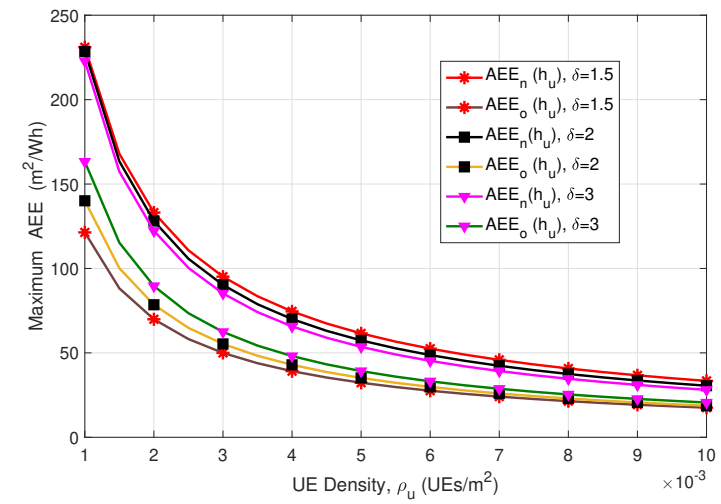

Fig. 4: Maximum AEE versus UE density: $\gamma_{\mathrm{n}}=6$.

to the increase in the number of the UEs in the coverage area; this increases the energy consumption (the denominator of the AEE) thereby reducing the respective maximum AEE values. The figure has three sets of plots, each corresponding to a particular power-reduction factor, $\delta$. For each set, the maximum AEE value achieved through the NOMA scheme prevails over the corresponding value of the OMA scheme. Also, the margin between maximum AEE values decreases with an increase in the user density. Furthermore, the gain in the AEE value achieved using the NOMA scheme, over the OMA scheme, decreases with the increase in the $\delta$ value. From the above discussions, it is clear that the proposed NOMA scheme is more energy-efficient when the power-reduction factor associated with the edge-UE lying in the coverage region, $\delta^{N-1}$, is relatively small. The factor either increases with an increase in the number of UEs $\left(\rho_{\mathrm{u}}\right)$ or with the $\delta$ value. Hence, the proposed scheme is recommended to use with a low $\delta$ value in applications where the user density is small. The lower-bound of the $\delta$ value is decided by the ability of the SIC receiver to successively decode the messages using the diversity in power received from the UEs.

\section{CONClusion}

In this work, we determined the optimal hovering altitude of a UAS deployed for a non-orthogonal multiple access uplink transmission scenario. The necessary power diversity required at the SIC receiver was ensured through the uplink power-reduction scheme that forms the basis of the P-NOMA scheme. The optimization problem maximized the proposed area energy efficiency metric while satisfying the altitude and the QoS constraints. The AEE-performance of the UAS with the underlying P-NOMA scheme is compared to the OMA scheme. It is observed that the P-NOMA scheme outperforms the OMA scheme and the corresponding achievable gain depends on the UE density and the power-reduction factor. The gain is inversely proportional with regard to the UE density and the power-reduction factor. The downlink-AEE analysis considering multiple UAS is left as future work.

\section{ACKNOWLEDGEMENT}

This work is supported by the project PAINLESS which has received funding from the European Union's Horizon 2020 research and innovation programme under grant agreement No 812991.

\section{REFERENCES}

[1] K. B. Letaief, W. Chen, Y. Shi, J. Zhang, and Y. A. Zhang, "The Roadmap to 6G: AI Empowered Wireless Networks," IEEE Communications Magazine, vol. 57, no. 8, pp. 84-90, Aug. 2019

[2] Y. Chen, A. Bayesteh, Y. Wu, B. Ren, S. Kang, S. Sun, Q. Xiong, C. Qian, B. Yu, Z. Ding, S. Wang, S. Han, X. Hou, H. Lin, R. Visoz, and R. Razavi, "Toward the Standardization of Non-Orthogonal Multiple Access for Next Generation Wireless Networks," IEEE Communications Magazine, vol. 56, no. 3, pp. 19-27, Mar. 2018.

[3] A. Fotouhi, H. Qiang, M. Ding, M. Hassan, L. G. Giordano, A. GarciaRodriguez, and J. Yuan, "Survey on uav Cellular Communications: Practical Aspects, Standardization Advancements, Regulation, and Security Challenges,'IEEE Communications Surveys Tutorials, pp. 1-1, 2019.

[4] Maraqa O, Rajasekaran AS, Al-Ahmadi S, Yanikomeroglu H, Sait SM. A Survey of Rate-Optimal Power Domain NOMA with Enabling Technologies of Future Wireless Networks. IEEE Communications Surveys Tutorials. 2020 Aug 3;22(4):2192-235.

[5] M. F. Sohail, C. Y. Leow and S. Won, "Energy-Efficient Non-Orthogonal Multiple Access for UAV Communication System," in IEEE Transactions on Vehicular Technology, vol. 68, no. 11, pp. 10834-10845, Nov. 2019, doi: 10.1109/TVT.2019.2939186.

[6] X. Liu et al., "Placement and Power Allocation for NOMA-UAV Networks," in IEEE Wireless Communications Letters, vol. 8, no. 3, pp. 965-968, June 2019, doi: 10.1109/LWC.2019.2904034.

[7] J. Sun, Z. Wang and Q. Huang, "Cyclical NOMA Based UAV-Enabled Wireless Network," in IEEE Access, vol. 7, pp. 4248-4259, 2019, doi: 10.1109/ACCESS.2018.2888855.

[8] A. A. Nasir, H. D. Tuan, T. Q. Duong and H. V. Poor, "UAV-Enabled Communication Using NOMA," in IEEE Transactions on Communications, vol. 67, no. 7, pp. 5126-5138, July 2019, doi: 10.1109/TCOMM.2019.2906622.

[9] F. Cui, Y. Cai, Z. Qin, M. Zhao and G. Y. Li, "Multiple Access for Mobile-UAV Enabled Networks: Joint Trajectory Design and Resource Allocation," in IEEE Transactions on Communications, vol. 67, no. 7, pp. 4980-4994, July 2019, doi: 10.1109/TCOMM.2019.2910263.

[10] D. Hu, Q. Zhang, Q. Li and J. Qin, ”Joint Position, Decoding Order, and Power Allocation Optimization in UAV-Based NOMA Downlink Communications," in IEEE Systems Journal, vol. 14, no. 2, pp. 29492960, June 2020, doi: 10.1109/JSYST.2019.2940985.

[11] N. Zhao et al., "Joint Trajectory and Precoding Optimization for UAV-Assisted NOMA Networks," in IEEE Transactions on Communications, vol. 67, no. 5, pp. 3723-3735, May 2019, doi: 10.1109/TCOMM.2019.2895831.

[12] R. Duan, J. Wang, C. Jiang, H. Yao, Y. Ren and Y. Qian, ”Resource Allocation for Multi-UAV Aided IoT NOMA Uplink Transmission Systems," in IEEE Internet of Things Journal, vol. 6, no. 4, pp. 7025-7037, Aug. 2019, doi: 10.1109/JIOT.2019.2913473.

[13] X. Pang et al., "Uplink Precoding Optimization for NOMA CellularConnected UAV Networks," in IEEE Transactions on Communications, vol. 68, no. 2, pp. 1271-1283, Feb. 2020, doi: 10.1109/TCOMM.2019.2954136.

[14] $\mathrm{Mu}$, Guangchen. "Joint Beamforming and Power Allocation for Wireless Powered UAV-Assisted Cooperative NOMA systems." EURASIP Journal on Wireless Communications and Networking 2020.1 (2020): 1-14.

[15] 3GPP; Technical Specification Group Services and System Aspects; Unmanned Aerial System (UAS) support in 3GPP; Stage 1; Release 17

[16] Zhang N, Wang J, Kang G, Liu Y. Uplink Non Orthogonal Multiple Access in 5G Systems. IEEE Communications Letters. 2016 Jan $25 ; 20(3): 458-61$.

[17] H. V. Abeywickrama, B. A. Jayawickrama, Y. He and E. Dutkiewicz, "Comprehensive Energy Consumption Model for Unmanned Aerial Vehicles, Based on Empirical Studies of Battery Performance," in IEEE Access, vol. 6, pp. 58383-58394, 2018, doi: 10.1109/ACCESS.2018.2875040 
[18] N. Babu, K. Ntougias, C. B. Papadias and P. Popovski, "Energy Efficient Altitude Optimization of an Aerial Access Point," IEEE 31st PIMRC, London, United Kingdom, 2020, pp. 1-7, doi: 10.1109/PIMRC48278.2020.9217265.

[19] N. Babu, C. B. Papadias and P. Popovski, "Energy-Efficient 3D Deployment of Aerial Access Points in a UAV Communication System," in IEEE Communications Letters, doi: 10.1109/LCOMM.2020.3017559.

[20] H. He, S. Zhang, Y. Zeng and R. Zhang, "Joint Altitude and Beamwidth Optimization for UAV-Enabled Multiuser Communications," in IEEE Communications Letters, vol. 22, no. 2, pp. 344-347, Feb. 2018, doi: 10.1109/LCOMM.2017.2772254.

[21] A. Al-Hourani, S. Kandeepan and S. Lardner, "Optimal LAP Altitude for Maximum Coverage," in IEEE Wireless Communications Letters, vol. 3, no. 6, pp. 569-572, Dec. 2014, doi: 10.1109/LWC.2014.2342736.

[22] 3GPP, "Physical Layer Procedures,", TR 36.213, Sep. 2015, v 10.12 .0 